EPJ Web of Conferences 37, 01014 (2012)

DOI: 10.1051/epjconf/20123701014

(C) Owned by the authors, published by EDP Sciences, 2012

\title{
Molecular interpretation of the XYZ states
}

\author{
R. Molina ${ }^{\mathrm{a}}$, D. Gamermann, and E. Oset
}

Departamento de Física Teórica and IFIC, Centro Mixto Universidad de Valencia-CSIC, Institutos de Investigación de Paterna, Aptdo. 22085, 46071 Valencia, Spain

\begin{abstract}
We study the vector - vector system including all the possible channels with quantum numbers charm $=0$, strangeness $=0$ around the energy region of $4000 \mathrm{MeV}$. New states with hidden charm around $4000 \mathrm{MeV}$ have been discovered by the B factories. They are intriguingly close to the $D^{*} \bar{D}^{*}$ and $D_{s}^{*} \bar{D}_{s}^{*}$ thresholds and do not have the properties of the charmonium states. We study the possible formation of $D^{*} \bar{D}^{*}$ and $D_{s}^{*} \bar{D}_{s}^{*}$ bound states in the framework of the Hidden Gauge formalism and discuss some of the models that favor the molecular assumption of some XYZ states: The hidden gauge formalism, Heavy baryon Chiral Perturbation Theory and the compositeness condition of Weinberg.
\end{abstract}

\section{Introduction}

The B factories at SLAC, KEK and CESR that originally were constructed to test matter-antimatter asymetries or CP violation, within or beyond the standard model, have discovered a number of charm and hidden charm mesons that do not seem to have the properties of the charmonium states. Some of these states are collected in Table 1 in [1]. The first of these XYZ states is the X(3872), observed by the Belle collaboration as a narrow peak near $3872 \mathrm{MeV}$ in the $\pi^{+} \pi^{-} J / \psi$ invariant mass distribution. The mass of the $\mathrm{X}(3872)$ is very close to the $D \bar{D}^{*}$ threshold which suggest it could be a bound state made of $D$ and $\bar{D}^{*}$. Many theoretical studies testing this hypothesis have been made [2-10]. The decay $X(3872) \rightarrow \pi^{+} \pi^{-} \pi^{0} J / \psi$ has been also observed and Belle quoted it to have the same ratio than to $\pi^{+} \pi^{-} J / \psi$, where the $3 \pi$ or $2 \pi$ come from $\omega$ or $\rho$, which in principle would imply a mixture of $I=0$ and $I=1$ [6]. However, an alternative explanation is given in [9]. Even though the X(3872) has mostly $I=0$, and both components, $D^{0} \bar{D}^{* 0}$ and $D^{+} \bar{D}^{*+}$ are needed in the same quantity to have isospin zero, it is the large $\rho$-width that makes the state to decay into $\pi^{+} \pi^{-} J / \psi$ for the lower components of the $\rho$-mass distribution. And thus, by taking into account the $\rho$ width in the calculation of the ratio $\frac{\Gamma(X(3872) \rightarrow J / \psi \omega)}{\Gamma(X(3872) \rightarrow J / \psi \rho)}$, D. Gamermann and E. Oset are able to explain the experimental value of this within the model. The states that we consider here are the X(3940), the Y(3940), the $\mathrm{X}(4160)$ and the $\mathrm{Z}(3930)$ and these are some of their common properties: 1) they are close to the $D^{*} \bar{D}^{*}$ or $D^{*} \bar{D}_{s}$ thresholds, 2) they have C-parity $=+, 3$ ) they are relatively narrow, 4) the estimated partial decay widths to $\omega J / \psi$ or $\phi J / \psi$ usually are above $1 \mathrm{MeV}$, which is quite larger than the measured partial decay widths for any of the observed hadronic transitions between charmonium states and 5) they have been observed in $B$ decays or in double charmonium production reaction $e^{+} e^{-} \rightarrow J / \psi+X$.

There are some theoretical approaches. In [11], the authors solved the Schrödinger equation from a potential that includes vector, pion and $\sigma$ exchange and they find molecualar solutions for the $\mathrm{Y}(3930)$ and $\mathrm{Y}(4140)$ with $J^{P}=0^{+}, 2^{+}$, concluding that they are molecular partners. In [12] the decay rates of the $Y(3940) \rightarrow J / \psi \omega$ and $Y(4140) \rightarrow J / \psi \phi$ are calculated under the assumption of hadronic molecuels with $J^{P C}=0^{++}$and they turn out to be consistent with the experimental data, which supports the molecular assumption. The coupling constants are calculated by means of the compositeness condition of Weinberg. In [13] it is argued that the peak at $4140 \mathrm{MeV}$ named as the $\mathrm{Y}(4140)$ is just a $\phi J / \psi$ threshold effect. In [14], the hidden gauge formalism is applied to study the vector - vector interaction in the charm sector

a e-mail: molina@rcnp.osaka-u.ac.jp

This is an Open Access article distributed under the terms of the Creative Commons Attribution License 2.0, which permits unrestricted use, distribution, and reproduction in any medium, provided the original work is properly cited. 
and the authors obtain dynamically generated states from this interaction which are close to the $D^{*} \bar{D}^{*}$ and $D_{s}^{*} \bar{D}_{s}^{*}$ thresholds. Some of them with quantum numbers, masses and widths compatible with the Z(3930), $\mathrm{Y}(3940)$ and $\mathrm{X}(4160)$.

\section{The hidden gauge formalism}

The HGS formalism to deal with vector mesons $[15,16]$ is a useful and internally consistent scheme which preserves chiral symmetry. In this formalism the vector meson fields are gauge bosons of a hidden local symmetry transforming inhomogeneously. After taking the unitary gauge, the vector meson fields transform exactly in the manner as in the non linear realization of chiral symmetry [17].

Following Ref. [18] the Lagrangian involving pseudoscalar mesons, photons and vector mesons can be written as $\mathcal{L}=\mathcal{L}^{(2)}+\mathcal{L}_{I I I}$, with

$$
\begin{array}{r}
\mathcal{L}^{(2)}=\frac{1}{4} f^{2}\left\langle D_{\mu} U D^{\mu} U^{\dagger}+\chi U^{\dagger}+\chi^{\dagger} U\right\rangle \\
\mathcal{L}_{I I I}=-\frac{1}{4}\left\langle V_{\mu \nu} V^{\mu \nu}\right\rangle+\frac{1}{2} M_{V}^{2}\left\langle\left[V_{\mu}-\frac{i}{g} \Gamma_{\mu}\right]^{2}\right\rangle,
\end{array}
$$

where $\langle\ldots\rangle$ represents a trace over $S U(3)$ matrices. The covariant derivative is defined by $D_{\mu} U=\partial_{\mu} U-$ $i e Q A_{\mu} U+i e U Q A_{\mu}$, being $Q=\operatorname{diag}(2,-1,-1) / 3, e=-|e|$ the electron charge, and $A_{\mu}$ the photon field. The chiral matrix $U$ is given by $U=e^{i \sqrt{2} \phi / f}$, being $f$ the pion decay constant $(f=93 \mathrm{MeV})$. The $\phi$ and $V_{\mu}$ matrices are the usual SU(3) matrices containing the pseudoscalar mesons and vector mesons respectively that we extend to SU(4) in order to include the charm sector mesons. The terms with $\chi$ in $\mathcal{L}^{(2)}$ provide the mass term for the pseudoscalars. Some of the important features in this framework is that: 1) For four pseudoscalar meson fields, the $\mathcal{L}^{(2)}$ Lagrangian provides the well known chiral Lagrangian at lowest order, $\widetilde{\mathcal{L}}^{(2)}=\frac{1}{12 f^{2}}\left\langle\left[\phi, \partial_{\mu} \phi\right]^{2}+M \phi^{4}\right\rangle$., 2) The term $\mathcal{L}_{\gamma P P}$, that appears in $\widetilde{\mathcal{L}}^{(2)}$, gets cancelled with one with opposite sign and same structure from $\mathcal{L}_{I I I}$ such that ultimately the photon couples to the pseudoscalars via vector meson exchange, the basic feature of VMD, 3) The hidden gauge coupling constant $g$ is related to $f$ and the vector meson mass $\left(M_{V}\right)$ through $g=\frac{M_{V}}{2 f}$, which is one of the forms of the KSFR relation.

From $\mathcal{L}_{I I I}$, we obtain the four-vector and three-vector contact terms:

$$
\begin{array}{r}
\mathcal{L}_{I I I}^{(c)}=\frac{g^{2}}{2}\left\langle V_{\mu} V_{v} V^{\mu} V^{v}-V_{v} V_{\mu} V^{\mu} V^{v}\right\rangle \\
\mathcal{L}_{I I I}^{(3 V)}=i g\left\langle\left(\partial_{\mu} V_{v}-\partial_{\nu} V_{\mu}\right) V^{\mu} V^{v}\right\rangle
\end{array}
$$

By means of Eq. (4), the vector exchange diagrams in Fig. 1 are calculated. The diagram in Fig. 1d) leads to a repulsive $\mathrm{p}$-wave interaction for equal masses of the vectors [19] and only to a minor component of swave in the case of different masses [20]. Thus, the four-vector-contact term in Fig. 1a) and the t(u)-channel vector exchange diagrams in Fig. 1c) are responsible for the generation of resonances or bound states if the interaction is strong enough. As we are dealing with vector mesons, the vector-vector dynamically generated states decay to pseudoscalar mesons apart from other pair of vector mesons whose threshold is below the mass of the state. We introduce the s-wave, spin and isospin projected amplitudes (contact + vector-exchange + (2Pseud.)-box diagrams) as the kernel V in the Bethe Salpeter equation:

$$
T=(\hat{1}-V G)^{-1} V
$$

where $G$ is the loop function. Therefore, we are summing all the diagrams containing zero, one, two... loops implicit in the Bethe Salpeter equation. Finally, we look for poles of the unitary $T$ matrix in the second Riemann sheet. All this procedure is well explained in $[19,20]$. It is worth to note that, first, the freedom in the model comes mainly from the parameter used to regularize the two-meson-function loop $G$ in Eq. (5), if dimensional regularization is used to compute it, the parameter will be the subtraction constant, whereas if the cutoff method is preferred, then, the tunning of the corresponding cutoff in the integral of the loop 
function will provide the mass of the states. Thus, only one parameter, $\alpha_{H}$, is needed to get the masses of the XYZ states considered here. Second, the SU(4) symmetry is strongly broken by the explicit use of the masses of the vector mesons in the vector-exchange diagrams, being the terms of the potential that come from the diagrams where a $D^{*}$ meson is exchanged proportional to $\kappa=\frac{m_{\rho}^{2}}{m_{D^{*}}^{2}} \sim 0.15$, which gives rise to corrections of the order of $10 \%$ of the $\rho$-exchange terms.

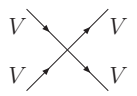

a)

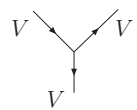

b)

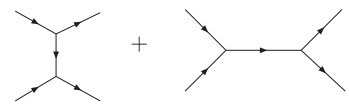

d)
Fig. 1. Terms of the $\mathcal{L}_{I I I}$ Lagrangian: a) four vector contact term, Eq. (3); b) three-vector interaction, Eq. (4); c) $t$ and $u$ channels from vector exchange; d) $s$ channel for vector exchange.

\section{The XYZ particles}

In view of the results obtained in the cases of the vector-vector interaction in SU(3) and the $\rho(\omega) D^{*}$ system in [21] we make an attempt to study now the sector with $C=0$ and $S=0$. In the case of $I=0$ we have 10 channels: $D^{*} \bar{D}^{*}, D_{s}^{*} \bar{D}_{s}^{*}, K^{*} \bar{K}^{*}, \rho \rho, \omega \omega, \phi \phi, J / \psi J / \psi, \omega J / \psi, \phi J / \psi$ and $\omega \phi$, and 6 channels for $I=1: D^{*} \bar{D}^{*}$, $K^{*} \bar{K}^{*}, \rho \rho, \rho \omega, \rho J \psi$ and $\rho \phi$. The function loop is computed by means of dimensional regularization and we fix the value of $\mu=1000 \mathrm{MeV}$ as in the work of [20]. We establish two different subtraction constants, one for the channels involving two light vector meson, which is called as $\alpha_{L}$, and the other one, for the channels involving two heavy vector meson: $\alpha_{H}$. When one channel involves both kind of mesons, one heavy and one light vector meson, we also put $\alpha_{L}$. The value of $\alpha_{L}$ is set to get the position of the pole $f_{2}(1270)$ as $\alpha_{L}=-1.65$, like it was done in the work of [20]. Thus, we get the correct position of the poles found in this work. The $\alpha_{H}$ constant is set to -2.07 in order to get the position of the pole found in $S=0$ around $3940 \mathrm{MeV}$. Note that, the tunning of the $\alpha_{L}$ parameter barely affect to the position of the $\mathrm{XYZ}$ states, being the important parameter $\alpha_{H}$, the one used in the heavy-heavy channels. By the fact of having several channels, ones involving heavy mesons and the other light mesons, the $g$ parameter in the Lagrangian should be different for the different cases. Thus, we put $g=M_{\rho} /\left(2 f_{\pi}\right)=4.17$ for light mesons, and $g_{D}=M_{D^{*}} /\left(2 f_{D}\right)=6.9, g_{D_{s}}=M_{D_{s}^{*}} /\left(2 f_{D_{s}}\right)=5.47, g_{\eta_{c}}=M_{J / \psi} /\left(2 f_{\eta_{c}}\right)=5.2$, when one $D^{*}, D_{s}^{*}$ or $J / \psi$ meson is involved. The $D \bar{D}$ decay mode is also considered by means of a box diagram as it was done in [21], but the effect is small compared with the (light) vector-(light or heavy) vector decay modes. The results for the pole position obtained together with a possible assignment with some of the XYZ particles observed in the energy region of $4000 \mathrm{MeV}$ are given in Table 1 in comparison with the experiment [1].

As it is shown in Table 1, we find one pole with a mass $\sim 3940 \mathrm{MeV}$ for each spin $J=0,1$ and 2 and $I=0$. The modules of the coupling contants to the different channels are obtained from the residues of the amplitudes and they are given in Tables 2 and 3. In Table 1 in [1], there are also three states around this mass. The state that we found for $I=1$ is discarded to be associated with one of these states, because in our model this state decays to $D \bar{D}$ and not to $\omega J / \psi$, thus cannot be associated with the $\mathrm{X}(3940)$, since the $D \bar{D}$ decay has not been observed [22], whereas it cannot be associated to the Y(3940) either, because the experimental state decays to $\omega J / \psi$. On the other hand, we find a width so large that it is likely to be assigned to the $\mathrm{Z}(3930)$. In that way, the state found for $I=1$ is a prediction of the model. In Table 1 we call it as $Y_{p}(3912)$, where ' $p$ ' stands for prediction. The state that we found for $J=1$ is also a prediction since it has C-parity negative. We can evaluate straightforwardly $\Gamma\left(\left(3943,0^{+}\left[0^{++}\right]\right) \rightarrow \omega J / \psi\right)$ from the model taking the coupling $\left|g_{Y \omega J / \psi}\right|=1445 \mathrm{MeV}$ from Table 2:

$$
\Gamma\left(\left(3943,0^{+}\left[0^{++}\right]\right) \rightarrow \omega J / \psi\right)=\frac{p\left|g_{Y \omega J / \psi}\right|^{2}}{8 \pi M_{Y}^{2}}
$$

where $p$ is the momentum of $\omega$ in the resonance rest frame. We obtain $\Gamma\left(\left(3943,0^{+}\left[0^{++}\right]\right) \rightarrow \omega J / \psi\right)=1.52$ $\mathrm{MeV}$, compatible with the expected experimental value for this decay, $\Gamma(Y(3940) \rightarrow \omega J / \psi)>1 \mathrm{MeV}[1]$. 
Table 1. Comparison of the mass, width and quantum numbers with the experiment. All the quantities are in units of $\mathrm{MeV}$.

\begin{tabular}{llllll}
\hline$I^{G}\left[J^{P C}\right]$ & Theory & \multicolumn{3}{c}{ Experiment } \\
\hline & (Mass, Width) & Name & Mass & Width & $J^{P C}$ \\
$0^{+}\left[0^{++}\right]$ & $(3943,17)$ & $Y(3940)$ & $3943 \pm 17$ & $87 \pm 34[30]$ & $J^{P+}$ \\
& & & $3914.3_{-3.4}^{+3.8} \pm 2$ & $34_{-8}^{+12} \pm 5[31]$ & \\
& & $3915 \pm 3 \pm 2$ & $17 \pm 10 \pm 3[32]$ & \\
$0^{-}\left[1^{+-}\right]$ & $(3945,0)$ & $" Y_{p}(3945) ”$ & & & \\
$0^{+}\left[2^{++}\right]$ & $(3922,55)$ & $Z(3930)$ & $3929 \pm 5$ & $29 \pm 10$ & $2^{++}$ \\
$0^{+}\left[2^{++}\right]$ & $(4157,102)$ & $X(4160)$ & $4156 \pm 29$ & $139_{-65}^{+113}$ & $J^{P+}$ \\
$1^{-}\left[2^{++}\right]$ & $(3912,120)$ & $" Y_{p}(3912) "$ & & & \\
\hline
\end{tabular}

Table 2. Quantum numbers, pole positions and modules of the couplings $\left|g_{i}\right|$ in units of MeV for $I=0$.

\begin{tabular}{llllllllllll}
\hline$I^{G}\left[J^{P C}\right]$ & $\sqrt{s}_{\text {pole }}$ & $D^{*} \bar{D}^{*}$ & $D_{s}^{*} \bar{D}_{s}^{*}$ & $K^{*} \bar{K}^{*}$ & $\rho \rho$ & $\omega \omega$ & $\phi \phi$ & $J / \psi J / \psi$ & $\omega J / \psi$ & $\phi J / \psi$ & $\omega \phi$ \\
\hline $0^{+}\left[0^{++}\right]$ & $3943+i 7.4$ & 18822 & 8645 & 15 & 52 & 1368 & 1011 & 422 & 1445 & 910 & 240 \\
$0^{-}\left[1^{+-}\right]$ & $3945+i 0$ & 18489 & 8763 & 40 & 0 & 0 & 0 & 0 & 0 & 0 & 0 \\
$0^{+}\left[2^{++}\right]$ & $3922+i 26$ & 21177 & 6990 & 44 & 84 & 2397 & 1999 & 1794 & 2433 & 3061 & 789 \\
$0^{+}\left[2^{++}\right]$ & $4169+i 66$ & 1319 & 19717 & 87 & 73 & 2441 & 3130 & 2841 & 2885 & 5778 & 1828 \\
\hline
\end{tabular}

Table 3. Quantum numbers, pole position and modules of the couplings $\left|g_{i}\right|$ in units of MeV for $I=1$.

\begin{tabular}{llllllll}
\hline$I^{G}\left[J^{P C}\right]$ & $\sqrt{s}_{\text {pole }}$ & $D^{*} \bar{D}^{*}$ & $K^{*} \bar{K}^{*}$ & $\rho \rho$ & $\rho \omega$ & $\rho J / \psi$ & $\rho \phi$ \\
\hline $1^{-}\left[2^{++}\right]$ & $3919+i 74$ & 20869 & 152 & 0 & 3656 & 6338 & 2731 \\
\hline
\end{tabular}

Then, we find a natural explanation on why this rate is much larger than it would be if it corresponded to a hadronic transition between charmonium states. On the other hand, the two states that we find in $I=0$ for $J=2$, with masses $M=3922$ and $4157 \mathrm{MeV}$, are assigned to the $\mathrm{Z}(3930)$ and $\mathrm{X}(4160)$ respectively, by the proximity of the mass, width and quantum numbers. The structure of the state found with $M=4157 \mathrm{MeV}$ is radically different to the other states, being the most important channel $D_{s}^{*} \bar{D}_{s}^{*}$.

\section{Heavy Hadron Chiral perturbation theory for heavy - heavy meson molecules}

One also could think in the forces between the two heavy meson inside the molecule in analogy with the deuteron . From this point of view, it is possible to formulate an effective field theory (EFT) for the description of the heavy meson molecules at low energies. As in the deuteron, the low energies interaction between the pair of heavy mesons is mediated by the exchange of pions. On the other hand, the short range interactions, which are unknown in principle, can be parametrized in terms of contact - range operators. Nevertheless, pions can be treated perturbatively in a larger range of energies than in the case of the nucleon - nucleon system which symplifies the EFT description of heavy meson molecules to a contact range theory. In Ref. [23], the authors start from the most general Lagrangian consistent with chiral symmetry for pions, isospin and spin symmetries for the heavy quark. At leading order in the EFT expansion, this Lagrangian contains two terms:

$$
\mathcal{L}=\mathcal{L}_{4 H}^{(0)}+\mathcal{L}_{\pi H H}^{(0)}
$$

where the first one contains a four meson interaction vertex and the second, the meson pion vertex [24],[25]. Thus, the s-wave leading order interactions (LO) between a heavy meson and an antimeson only contains contact operators. The contact range interactions are constraint by HQSS and mix different particle channels 
with the same quantum numbers: $\left(0^{++}\right) \rightarrow P P, P^{*} P^{*}, 1^{+-} \rightarrow \frac{1}{\sqrt{2}}\left(P \bar{P}^{*}+P^{*} \bar{P}\right), P^{*} \bar{P}^{*},\left(1^{++}\right) \rightarrow \frac{1}{\sqrt{2}}\left(P \bar{P}^{*}-P^{*} \bar{P}\right)$, $2^{++} \rightarrow P^{*} \bar{P}^{*}$. In this basis, the EFT potential is independent of momentum [23]. In this framework, the potential for $1^{++}$and $2^{++}$are identical, which is a hint for a $2^{++} D^{*} \bar{D}^{*}$ spin partner of the $\mathrm{X}(3872)$. When looking for poles, the authors apply the bound state equation and go to the second Riemann sheet of the complex plane of the energy. The EFT potential can be regularized with a cutoff $\Lambda$ and set the value of $C_{0}(\Lambda)$ to reproduce the position of a bound state. One important point, is that the authors redo the calculation without including the pion exchange interaction and conclude that $\mathrm{D}$-wave probabilities, expected to be a second order correction in the theory, are very small $1-4 \%$. In addition, they also find that the effect of considering coupled channels instead of a single channel $\left(D \bar{D}, D^{*} \bar{D}^{*}\right.$ or $\left.D^{*} \bar{D}\right)$ has also small effects, being suppressed by two orders in the EFT expansion. Thus, the binding energies of the $0^{++}$and $1^{+-}$states scales as $\left|\Delta E_{B}\right| \simeq\left|E_{B}\right|\left(\frac{\gamma_{B}}{\Lambda_{C}}\right)^{2}$, being $\gamma_{B}=\sqrt{-2 \mu E_{B}}$. As a conclusion of the work, the identification of the $\mathrm{X}(3872)$ and $\mathrm{X}(3915)$ resonances as isoscalar $D \bar{D}^{*}$ and $D^{*} \bar{D}^{*}$ molecules, leads in this model to the existence of four molecular partners of these two states. These four predicted bound states are named as $\mathrm{X}(3700)(D \bar{D}$, $\left.J^{P C}=0^{++}\right), \mathrm{X}(3814)\left(D^{*} \bar{D}, J^{P C}=1^{+-}\right), \mathrm{X}(3950)\left(D^{*} \bar{D}^{*}, J^{P C}=1^{+-}\right)$and $\mathrm{X}(4012)\left(D^{*} \bar{D}^{*}, 2^{++}\right)$, where the inputs are the masses of the $\mathrm{X}(3872)\left(D^{*} \bar{D}, 1^{++}\right)$and $\mathrm{X}(3915)\left(D^{*} \bar{D}^{*}, 0^{++}\right)$. Note that within the hidden gauge formalism described in the previous section, in [26], [9], the authors found before the $X(3700)$, the $\mathrm{X}(3872)$ with $J^{P C}=1^{++}$and another state with mass $=3875 \mathrm{MeV}$ and $J^{P C}=1^{+-}$and for the the $D^{*} \bar{D}^{*}$ states described in the previous section, the states found in [14] with $J^{P C}=0^{++}, 1^{+-}$have very similar properties to those in [23], where the discrepancies come for the state with $J^{P C}=2^{++}$, that these authors found at higher energies. The similar results of the two different calulations is a hint in favor of the molecular assumption of these XYZ states and the observation of the theoretical state X(3700).

\section{Compositeness condition for bound states}

One of the methods very useful to identify hadronic molecules in the spectrum is based on the compositeness condition for bound states of Weinberg [27]. Let us consider a two-body scattering system with one bound state in a nonrelativistic field theory. One can decompose the full hamiltonian $\mathcal{H}$ into the free part $\mathcal{H}_{0}$ and the interaction $V$ and assume that the Hilbert space of $\mathcal{H}_{0}$ consists of the bare bound state $\left|B_{0}\right\rangle$ and the two-body scattering states, i. e., $1=\left|B_{0}\right\rangle\left\langle B_{0}\left|+\int d \mathbf{k}\right| \mathbf{k}\right\rangle\langle\mathbf{k}|$. In the same way, eigenstates of the full hamiltonian $\mathcal{H}$ are given by the bound state $\left|B_{0}\right\rangle$ with the binding energy $B>0$ and the continuum states as $\mid \mathbf{k}$, full $\rangle\langle\mathbf{k}$, full $|$. Now we define the field renormalization constant $Z$ as the overlap of the bare state $\left|B_{0}\right\rangle$ and the physical bound state as $Z \equiv\left|\left\langle B_{0} \mid B\right\rangle\right|^{2}$, which takes a value $0 \leq Z \leq 1$. Since $\left|B_{0}\right\rangle$ is considered to be an elementary particle, $Z$ expresses the degree of "elementary" of the physical state. The quantity $1-Z$ can then be interpreted as the "compositeness" of the physical state. On the other hand, Low's scattering equation can be obtained by using the formal solution of the Lippmann-Schwinger equation and the complete set of the full Hamiltonian as [27]. In the limit that the binding energy $B$ is much smaller than the inverse of the range of forces [28], the compositeness can be expressed in terms of binding energy and coupling constant $g_{W} \equiv G_{W}(E=-B)$ with $G_{W}(E) \equiv\langle\mathbf{k}|V| B\rangle$ the vertex form factor,

$$
1-Z \sim 2 \pi^{2} \sqrt{2 \mu^{3}} \frac{g_{W}^{2}}{\sqrt{B}}
$$

Note also that this equation is an approximated form valid only for the small $B$, but the result is model independent because the explicit form of the interaction $V$ is not relevant. All the information of the interaction is embedded in the binding energy and coupling constant.

The method was already applied in [29], where the authors calculated the invariant mass spectrum for $\psi^{\prime} \pi^{+} \pi^{-}$as well as the corresponding $\pi \pi$ and $K \bar{K}$ spectra in the mass range of the $\mathrm{Y}(4660)$ is an $f_{0}(980) \psi^{\prime}$ bound state. A very good description of both spectra where data exist was achieved. In addition, the compositeness condition for bound states is applied in [12], where the authors suppose that the Y(3940) and $\mathrm{Y}(4140)$ are $D^{*} \bar{D}^{*}$ and $D_{s}^{*} \bar{D}_{s}^{*}$ bound states respectively with $J^{P C}=0^{++}$, calculate the couplings to their constituents by means of the compositeness condition and with these, they can evaluate $J / \psi V(V=\omega, \phi)$ decay widths which finally support this interpretation when comparing with the experiment. 


\section{Conclusions}

We have studied the $C=0$ and $S=0$ sector around the energy region of $4000 \mathrm{MeV}$ within the hidden gauge formalism combined with coupled channel unitarity. We find one state with mass $\sim 3940 \mathrm{MeV}$ for each spin $J=0,1$ and 2 . Only the states with $J=0$ and 2 can be associated the XYZ particles with mass $\sim 3940 \mathrm{MeV}$ in the PDG. Concretely, we associate the $J^{P C}=0^{++}$state with the $\mathrm{Y}(3940)$, and the $2^{++}$state with $\mathrm{Z}(3930)$. The $I=0 ; J^{P C}=1^{+-}$and $I=1 ; J^{P C}=2^{++}$are predictions of the model. Our model predicts another state with mass around $4160 \mathrm{MeV}$ that can be associated with the X(4160), being the $D *_{s} \bar{D}_{s}^{*}$ channel the most important channel. We have compared the results of the hidden gauge formalism with those of the HBChPT, two different formalisms that are giving similar results, what would imply a dominant potential from the $\rho, \omega$ exchange and less important for the $\pi$ exchange. In addition, the compositeness condition of Weinberg gives us couplings of the states to the $D^{*} \bar{D}^{*}$ and $D_{s}^{*} \bar{D}_{s}^{*}$ component in agreement with the ones found in the hidden gauge formalism for the $\mathrm{X}(3700), \mathrm{X}(3872), \mathrm{XYZ} \sim 3940$ and $\mathrm{X}(4160)$ as $D_{(s)}^{(*)} \bar{D}_{(s)}^{(*)}$ molecules.

\section{References}

1. S. Godfrey and S. L. Olsen, Ann. Rev. Nucl. Part. Sci. 58, 51 (2008)

2. M. B. Voloshin, L. B. Okun, JETP Lett. 23, 333 (1976) [Pisma Zh. Eksp. Teor. Fiz. 23, 369 (1976)].

3. A. De Rujula, H. Georgi, S.L. Glashow, Phys. Rev. Lett. 38, 317 (1976)

4. N. A. Tornqvist, Z. Phys. C 61, 525 (1994)

5. D. Gamermann and E. Oset, Eur. Phys. J. A 33, 119 (2007)

6. F. E. Close and P. R. Page, Phys. Lett. B 578, 119 (2004)

7. E. S. Swanson, Phys. Lett. B 588, 189 (2004)

8. E. Braaten and M. Kusunoki, Phys. Rev. D 72, 054022 (2005)

9. D. Gamermann and E. Oset, arXiv:0905.0402 [hep-ph].

10. M. B. Voloshin, Phys. Lett. B 579, 316 (2004) T. Barnes, S. Godfrey and E. S. Swanson, Phys. Rev. D 72, $054026(2005)$

11. X. Liu and Shi-Lin Zhu, Phys. Rev. D 80, 017502 (2009)

12. T. Branz, T. Gutsche and V. E. Lyubovitskij, Phys. Rev. D 80, 054019 (2009)

13. E. van Beveren, G. Rupp [arXiv:0906.2278v1 [hep-ph]]

14. R. Molina and E. Oset, Phys. Rev. D 80, 114013 (2009)

15. M. Bando, T. Kugo, S. Uehara, K. Yamawaki and T. Yanagida, Phys. Rev. Lett. 54, 1215 (1985).

16. M. Bando, T. Kugo and K. Yamawaki, Phys. Rept. 164 (1988) 217.

17. S. Weinberg, Phys. Rev. 166, 1568 (1968).

18. G. Ecker, J. Gasser, H. Leutwyler, A. Pich and E. de Rafael, Phys. Lett. B 223, 425 (1989).

19. R. Molina, D. Nicmorus and E. Oset, Phys. Rev. D 78, 114018 (2008)

20. L. S. Geng and E. Oset, Phys. Rev. D 79, 074009 (2009).

21. R. Molina, H. Nagahiro, A. Hosaka and E. Oset, Phys. Rev. D 80, 014025 (2009)

22. K. Abe et al., Phys. Rev. Lett. 98, 082001 (2007). P. Pakhlov et al. [Belle Collaboration], Phys. Rev. Lett. 100, 202001 (2008)

23. J. Nieves and M. P. Valderrama, arXiv:1204.2790 [hep-ph].

24. M. T. AlFiky, F. Gabbiani and A. A. Petrov, Phys. Lett. B 640, 238 (2006)

25. B. Grinstein, E. E. Jenkins, A. V. Manohar, M. J. Savage and M. B. Wise, Nucl. Phys. B 380, 369 (1992)

26. D. Gamermann, E. Oset, D. Strottman and M. J. Vicente Vacas, Phys. Rev. D 76, 074016 (2007)

27. S. Weinberg, Phys. Rev. 137, B672 (1965)

28. V. Baru, J. Haidenbauer, C. Hanhart, Yu. Kalashnikova and A. E. Kudryavtsev, Phys. Lett. B 586, 53 (2004).

29. F.-K. Guo, C. Hanhart and U.-G. Meißner, Phys. Lett. B 665, 26 (2008)

30. K. Abe et al. [Belle Collaboration], Phys. Rev. Lett. 94, 182002 (2005).

31. B. Aubert et al. [BaBar Collaboration], Phys. Rev. Lett. 101, 082001 (2008)

32. S. Uehara et al. [Belle Collaboration], Phys. Rev. Lett. 104, 092001 (2010) 